\title{
Reencuentro de Iluocoetes facali Lloris \& Rucabado, 1987 (Perciformes: Zoarcidae) en el sector norte del archipiélago patagónico occidental
}

\author{
Encounter of Iluocoetes facali Lloris \& Rucabado, 1987 (Perciformes: Zoarcidae) \\ in north western Patagonian archipelago \\ Pablo R. Reyes ${ }^{1}$ \\ ${ }^{1}$ Falkland Islands Fisheries Department. P.O. Box 598, F.I.P.A.S.S. Stanley, Falkland Island, U.K. \\ preyes@fisheries.gov.fk
}

\begin{abstract}
The first record of the species Iluocoetes facali Lloris \& Rucabado, 1987 in Chilean waters is given, extending its northern distribution in the western South American Cone in more than 2,500 km. Morphometrics and meristic characteristics of the specimen caught in intertidal waters of the Putemun Fjord $\left(42^{\circ} 28^{\prime} \mathrm{S}-73^{\circ} 51^{\prime} \mathrm{S}\right)$, located 15 $\mathrm{km}$ north of Castro, Chiloé Island, is given. This one is the second specimen known of the species after its description.
\end{abstract}

Additionally, in the present note, a taxonomic key to recognize the Chilean species of Iluocoetes and photographic material that allows corroborating the new record are included.

Key words: Chile, Chiloé, Ilucoetes fimbriatus, Ilucoetes elongatum, ichthyogeography

a la existencia de la especie (Anderson \& Fedorov 2004).

En las costas de Chile, según Pequeño (1989), el género Iluocoetes Jenyns, 1842, solamente estaba representado por dos especies: I. fimbriatus Jenyns, 1842 e I. elongatum (Smitt, 1898). En 1987, en la zona intermareal de Puerto Harberton, vertiente norte del

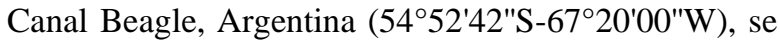
registró el primer y único ejemplar conocido de la especie I. facali, la cual fue descrita por Lloris \& Rucabado (1987). La cita de esta especie provocó controversia por parte de Anderson \& Gosztonyi (1991), quienes apuntan a que el holotipo correspondería a un ejemplar juvenil de Iluocoetes fimbriatus, y por lo tanto sugieren adscribirla a este binomio.

Tras el estudio de diversos ejemplares juveniles y adultos de I. fimbriatus procedentes del Museo Nacional de Historia Natural de París, Francia y de la Fisheries Research Station de la Universidad de Japón, Lloris \& Rucabado (1991) confirman la validez del binomio I. facali. Con el devenir de los años no se han registrado nuevas citas de I. facali, lo cual volvió a generar incertidumbre en el mundo científico respecto
El presente estudio tiene como objetivo corroborar la validez de la especie de acuerdo con lo descrito por Lloris \& Rucabado (1987), en base a material recolectado en la Isla de Chiloé y depositado en el Museo Nacional de Historia Natural de Chile. A partir de este antecedente se entrega el primer registro de $I$. facali en Chile y, por lo tanto, para el Océano Pacífico suroriental, el cual permite ampliar en $2.500 \mathrm{~km}$ la distribución septentrional de la especie.

\section{Material y métodos}

El material estudiado proviene de la colección de peces del Museo Nacional de Historia Natural de Chile. El espécimen de 144,6 mm de longitud total se encontró depositado en la sección de Hidrobiología, sin número de catálogo y con un único rótulo que consignaba su captura en el sector intermareal del fiordo Putemún de Castro, Isla de Chiloé. Si bien el colector se mantiene en el anonimato por falta de información, la fecha de captura del ejemplar puede ser calculada a antigua data, que se deberá remontar al menos 20 años (1975) 
Tabla 1

Morfometría (en porcentaje de longitud total) y merística comparada de Iluocoetes facali con ejemplares de I. fimbriatus. La nomenclatura de las abreviaciones se entrega en Material y métodos

Compared morphometrics (in percentage of total length) and meristics parameters between Iluocoetes facali and I. fimbriatus. The nomenclature abbreviations is given in Material and methods

\begin{tabular}{|c|c|c|c|c|c|c|}
\hline & $\begin{array}{c}\text { I. facali } \\
\text { IIPB } \\
\text { 114/1987 }\end{array}$ & $\begin{array}{c}\text { I. facali } \\
\text { MNHNC-P } \\
7279 \\
\end{array}$ & $\begin{array}{c}\text { I. fimbriatus } \\
\text { IZUA-PM } \\
2345 \\
\end{array}$ & $\begin{array}{c}\text { I. fimbriatus } \\
\text { MNHNC-P } \\
7278\end{array}$ & $\begin{array}{c}\text { I. fimbriatus } \\
\text { MNHNC-P } \\
7277 \\
\end{array}$ & $\begin{array}{c}\text { I. fimbriatus } \\
\text { MNHNC-P } \\
7278\end{array}$ \\
\hline $\mathrm{LT}$ & - & 144,6 & 230 & 260 & 300 & 306 \\
\hline LE & 100 & 100 & 100 & 100 & 100 & 100 \\
\hline LC & 20,8 & 20,1 & 18,1 & 20,6 & 17,5 & 21,5 \\
\hline AMC & - & 11,5 & 7,0 & 7,7 & 12,8 & 11,2 \\
\hline $\mathrm{AC}$ & - & 13,1 & 8,8 & 9,4 & 9,8 & 9,5 \\
\hline AOP & - & 7,8 & 6,4 & 7,7 & 7,1 & 8,1 \\
\hline LR & 6,9 & 6,9 & 7,7 & 7,4 & 7,4 & 7,6 \\
\hline LMS & 9,9 & 7,9 & 6,6 & 6,9 & 6,0 & 6,8 \\
\hline LMI & - & 5,3 & 4,9 & 5,0 & 4,7 & 4,9 \\
\hline $\mathrm{AO}$ & - & 9,1 & 5,9 & 6,4 & 6,8 & 6,4 \\
\hline AIN & - & 4,4 & 3,7 & 3,8 & 4,9 & 4,3 \\
\hline PD & 20,8 & 20,4 & 18,6 & 23,9 & 19,6 & 21,5 \\
\hline LBAD & 82,2 & 86,1 & 70,6 & 75,8 & 76,1 & 74,4 \\
\hline PA & 42,6 & 43,0 & 33,7 & 41,7 & 40,5 & 41,5 \\
\hline LBA & 64,1 & 61,9 & 52,6 & 56,3 & 54,7 & 56,7 \\
\hline LPR & 21,8 & 19,8 & 17,6 & 21,5 & 21,1 & 20,7 \\
\hline LP & 10,9 & 12,4 & 9,8 & 11,2 & 11,9 & 11,6 \\
\hline LP1 & 4 & 4,1 & 3,2 & 3,4 & 2,4 & 3,3 \\
\hline LPP & - & 17,8 & 14,5 & 18,4 & 19,6 & 16,6 \\
\hline DO & 4 & 3,2 & 3,5 & 4,4 & 4,9 & 4,1 \\
\hline PRO & - & 6,5 & 7,7 & 7,6 & 7,4 & 7,6 \\
\hline IN & 3 & 5,3 & 2,3 & 2,9 & 5,6 & 3,6 \\
\hline $\mathrm{AM}$ & 10,9 & 16,0 & 8,9 & 11,9 & 13,4 & 12,1 \\
\hline $\mathrm{D}$ & 89 & 85 & 87 & 90 & 85 & 85 \\
\hline A & 76 & 74 & 71 & 72 & 71 & 73 \\
\hline $\mathrm{P}$ & 15 & 14 & 17 & 17 & 17 & 17 \\
\hline $\mathrm{V}$ & 3 & 3 & 3 & 3 & 3 & 3 \\
\hline BR & 12 & 11 & 11 & 12 & 12 & 12 \\
\hline BT & 5 & 5 & 5 & 5 & 5 & 5 \\
\hline PPO & 2 & 1 & 1 & 2 & 2 & 2 \\
\hline PSO & 2 & 2 & 2 & 2 & 2 & 2 \\
\hline SUB & 7 & 8 & 8 & 7 & 8 & 7 \\
\hline PPM & 8 & 7 & 7 & 7 & 7 & 8 \\
\hline
\end{tabular}

(A. Cornejo, com. pers.) ${ }^{1}$, lo cual sitúa la captura del ejemplar en una fecha anterior a la descripción de la especie. El ejemplar tras ser identificado siguiendo la literatura especializada (Anderson 1994, Lloris \& Rucabado 1991) fue ingresado a la colección de peces del Museo Nacional de Historia Natural de Chile con

\footnotetext{
${ }^{1}$ Cornejo, A. 2005. Sección de Hidrobiología. Museo Nacional de Historia Natural de Chile. Santiago de Chile.
}

el número MNHNC-P 7279. Las mediciones morfométricas fueron efectuadas con pie de metro digital a un nivel de precisión de mm. Los conteos merísticos fueron realizados con microscopio estereoscópico siguiendo la descripción y nomenclatura de poros cefálicos entregada por Anderson (1994) y Lloris \& Rucabado (1991). Como material comparativo se empleó la descripción del 
ejemplar I. facali IIPB 114/1987 efectuada por Lloris \& Rucabado (1991) y los ejemplares de I. fimbriatus IZUA-PM 2345, MNHNC-P 7278 (2 ejemplares) y MNHNC-P 7277; el primero de ellos fue recolectado en aguas internacionales del Atlántico suroriental

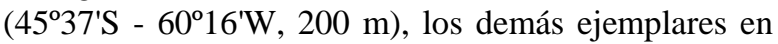
aguas del archipiélago patagónico occidental (Pacífico suroccidental). Las abreviaturas de los recuentos y porcentajes de las medidas utilizadas en el texto corresponden a: A radios aleta anal; $\mathrm{P}$ radios aleta pectoral; V radios aleta pélvica. Las siguientes unidades son tomadas como porcentaje de la longitud estándar; AC ancho máximo de la cabeza; AM altura mayor corporal; AMC altura mayor de la cabeza; AO ancho oral; AOP altura opérculo; AIN ancho internasal; BR branquispinas; BT branquióstegos; D radios aleta dorsal; DO diámetro de la órbita; IN ancho interorbital; LBA longitud base aleta anal; LC longitud de la cabeza; LE longitud estándar; LMS longitud mandíbula superior; LMI longitud mandíbula inferior; LP1 longitud de la aleta pélvica; LPP longitud prepélvica; LP longitud de la aleta pectoral; LR longitud rostro; LPR longitud prepectoral; LT Longitud total; PA longitud preanal; PD longitud predorsal; LBAD longitud base aleta dorsal; PRO longitud preorbitaria; SUB poros suborbitales; PPO poros postorbitales; PSO poros supraorbitales; PPM Poros Preopérculo-mandibulares. En la Tabla 1, el signo de guión (-) indica que la medición no fue efectuada al holotipo de I. facali (Lloris \& Rucabado 1987), pero sí al ejemplar MNHNC-P 7279, como manera de contribuir al conocimiento taxonómico de la especie.

\section{Resultados}

La descripción del ejemplar MNHNC-P 7279 coincide con la descripción del holotipo de I. facali (IIPB 114/1987), es decir: posee el cuerpo alargado y comprimido, sobre todo en la mitad posterior. Sus escamas están embebidas en la piel, son de aspecto lenticular, no muy abundantes en el cuerpo y más densamente presentes a lo largo de las bases de las aletas dorsal y anal. La cabeza es algo deprimida, desprovista de escamas y apéndices dérmicos. Los ojos son algo prominentes, sobresaliendo claramente del perfil superior cefálico, el diámetro ocular es igual a la longitud de la aleta ventral, algo mayor que la distancia interorbitaria. La boca es subterminal, con la comisura bucal en la vertical que pasa por el borde posterior del ojo. La mandíbula superior está algo proyectada hacia delante, de tal forma que la inferior se encaja en ella, permitiendo a su vez visualizar un par de dientes cónicos bien aparentes. Los labios forman un repliegue muy claro y visible. La membrana palatina está presente. Los dientes son cónicos y están presentes en las mandíbulas, vómer y palatinos. Los orificios nasales o narinas son tubulares. Con poros cefálicos dispuestos en series. Las aberturas branquiales se originan en la parte superior de la base de las aletas pectorales y finalizan justo en el lugar de inserción de las ventrales. Posee una sola aleta dorsal, confluente con la caudal y la anal, cuyo origen se sitúa en la vertical que pasa por la base de las pectorales. Las pectorales son pequeñas y con el borde algo festoneado. Las ventrales son iguales en su longitud al diámetro ocular y su extremo libre alcanza a la base inferior de las aletas pectorales (Fig. 1). La coloración general del cuerpo del ejemplar MNHNC-P 7279 bajo condiciones de preservación en formalina coincide con la del holotipo, es decir: con tendencia al pardo claro, casi rojizo. A lo largo del cuerpo y cola existe una serie de manchas vermiculares claras y oscuras que se extienden por la región ventral. La región mandibular y gular es claras. La aleta dorsal presenta una serie de manchas oscuras dobles más intensas en el borde de la misma que en la base de unión a lo largo del cuerpo. Finalmente, el peritoneo es negruzco.

La morfometría del ejemplar MNHNC-P 7279 resultó ser similar a la del holotipo. Existen variaciones morfométricas porcentuales de bajo orden entre el ejemplar holotipo y el ejemplar estudiado, particularmente en la longitud de la mandíbula superior $(9,9 \mathrm{~V} / \mathrm{S} 8,0)$, la longitud de la base de la aleta dorsal (82.2 V/S 86.1), longitud de la base de la aleta anal (64.1 V/S 61.9) y la altura mayor corporal (10,9 V/S 16,0). Los otros 17 caracteres estudiados presentaron una baja variabilidad. Los detalles de las mediciones morfométricas efectuadas al ejemplar MNHNC-P 7279 y su comparación con el holotipo se entregan en la Tabla 1.

La merística del ejemplar de I. facali MNHNC-P 7279 con su holotipo presentó un alto grado de similitud, sin embargo, algunos caracteres varían en un bajo orden de magnitud, entre ellos se encuentran; radios pectorales, anales y pélvicos, branquiespinas, branquióstegos, poros postorbitales, poros supraorbitales, poros suborbitales y poros preoperculomandibulares. Todos los caracteres se encuentran en un rango de variación esperable, que no supera la unidad. Estas leves diferencias merísticas entre 


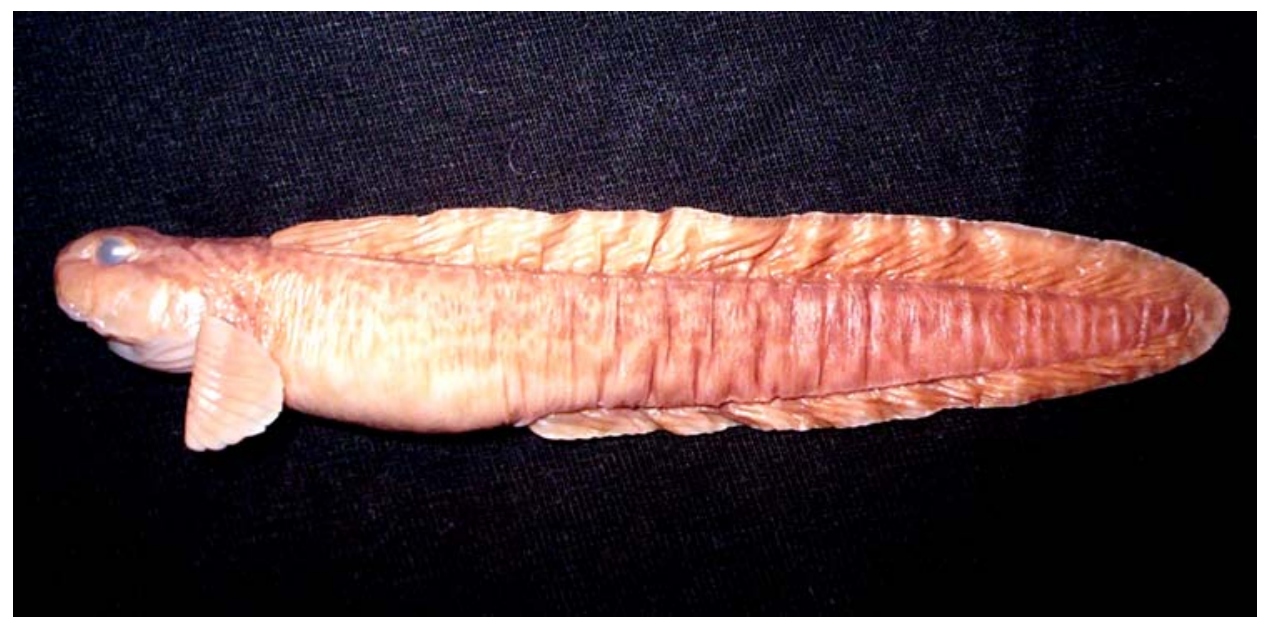

Figura 1

Ejemplar Iluocoetes facali Lloris \& Rucabado, 1987 MNHNC-P 7279, 144,6 mm de longitud total Specimen Iluocoetes facali Lloris \& Rucabado, 1987 MNHNC-P 7279, 144.6 mm total length

ejemplares de una misma especie que habitan en la región austral de Chile y en aguas de Chile centro sur son provocadas por un efecto de la latitud geográfica sobre su cuerpo, desencadenado por la temperatura del agua donde viven, tal efecto es llamado variación clinal, y ha sido ampliamente notificado en especies de peces que habitan en la costa de Chile (Lloris \& Rucabado 1991, Pequeño 1978).

\section{Discusión}

Al comparar la morfometría de los ejemplares de $I$. facali provenientes de Chiloé y el Canal Beagle, con ejemplares de I. fimbriatus provenientes del Atlántico suroriental y el Pacífico suroccidental (Tabla 1), se observa una serie de caracteres comunes explicando la confusión respecto al estatus taxonómico de $I$. facali. Sin embargo, en el presente estudio se identificaron cinco caracteres que presentan diferencias, éstos son la longitud del rostro $\operatorname{LR}(6,9 \mathrm{~V} / \mathrm{S} 7,5)$, la longitud de la mandíbula superior LMS (8,9 V/S 6,5), la longitud de la base de la aleta dorsal LBAD (84,2 V/S 74,2), la longitud de la base de la aleta anal LBA $(63 \mathrm{~V} / \mathrm{S} 55,1)$ y la longitud de las aletas pélvicas LP1 (4,1 V/S 3,1). Al comparar la merística de los ejemplares estudiados de ambas especies (Tabla 1) se observa una situación análoga a la descrita para la morfometría, existiendo dos caracteres que en sus promedios presentan claras diferencias, éstos son el número de radios de la aleta anal A (75 V/S 72) y el número de radios de la aleta pectoral P (15 V/S 17)

Resulta importante señalar que durante el desarrollo del proyecto Español-Argentino BIOICTIOS (19871988), fueron descritas tres nuevas especies de zoárcidos: Iluocoetes facali Lloris \& Rucabado, 1987; Crossostomus sobrali Lloris \& Rucabado, $1987 \mathrm{y}$ Pogonolycus marinae (Lloris, 1988), las cuales fueron cuestionadas como taxa, sugiriéndose la adscripción de todas ellas a juveniles de otras especies congenéricas (Anderson \& Gosztonyi 1991). Sin embargo, con el del tiempo esta situación ha sido lentamente resuelta. En el caso de $C$. sobrali, sabemos en la actualidad que su condición ha sido aclarada al dilucidarse su estatus taxonómico y validarse como especie, situación bien descrita en aguas chilenas por Morrison \& Pequeño (2003). A su vez, P. marinae ha sido reconocida como especie válida por los estudios que presentan mayor grado de actualización acerca de la familia Zoarcidae (Anderson \& Fedorov 2004). Con ello, la única especie descrita durante el desarrollo del proyecto BIOICTIOS cuya situación no había podido ser dilucidada era $I$. facali, principalmente por la ausencia de ejemplares en las colecciones ictiológicas y por el vacío existente en cuanto a registros; sin embargo, el análisis del ejemplar MNHNC-P 7279 ha permitido confirmar la existencia de $I$. facali, realizar la primera cita documentada de la especie tras casi veinte años sin registro, notificando su presencia en el sector norte del 
archipiélago patagónico occidental y ampliar su distribución geográfica en 2.500 km.

Con los antecedentes proporcionados por el presente estudio se confina la distribución geográfica de $I$. facali entre el canal Beagle (5452'S) y Chiloé (42 28 'S); por lo tanto, hasta la fecha la especie se encuentra circunscrita al litoral de canales y senos del archipiélago patagónico occidental, el cual a pesar de ser un ambiente bastante uniforme, presenta dos grandes unidades: una al norte de la península de Taitao (40 a $\left.44^{\circ} \mathrm{S}\right)$ y otra al sur de ésta (44 a $\left.56^{\circ} \mathrm{S}\right)$, siendo ambas unidades parte de la región biogeográfica latitudinal "sur fría-temperada” de la costa de Chile, cuyos límites se encuentran entre los 40 y $56^{\circ} \mathrm{S}$ (Ojeda et al. 2000) y definen la distribución geográfica de I. facali.

Finalmente, el género Iluocoetes queda conformado por tres especies en aguas chilenas: $I$. fimbriatus Jenyns, 1842, I. elongatum (Smitt, 1898) e I. facali Lloris \& Rucabado, 1987, que pueden reconocerse mediante la siguiente clave:

1 (2) Radios branquióstegos $4+2$ Iluocoetes elongatum

2 (1) Radios branquióstegos 3+2

3 (4) Longitud de la base de la aleta anal es mayor al 60\% de la LT. Longitud de la base de la aleta dorsal es mayor al $80 \%$ de la LT. Longitud del rostro es menor al 7\% de la LT. Longitud de la mandíbula superior es mayor al 7\% de la LT. Longitud de la aleta pélvica es mayor al $4 \%$ de la LT. Aleta pectoral con menos de 16 radios.

Iluocoetes facali

4 (3) Longitud de la base de la aleta anal es menor al 60\% de la LT. Longitud de la base de la aleta dorsal es menor al $80 \%$ de la LT. Longitud del rostro es mayor al 7\% de la LT. Longitud de la mandíbula superior es menor al 7\% de la LT. Longitud de la aleta pélvica es menor al 4\% de la LT. Aleta pectoral con más de 16 radios.

Iluocoetes fimbriatus

\section{Agradecimientos}

Al Dr. Domingo Lloris del Instituto de Ciencias del Mar (CMIMA-CSIC) de Barcelona, España, por sus comentarios. Al Dr. Germán Pequeño del Instituto de Zoología de la Universidad Austral de Chile por sus importantes aportes bibliográficos. Al Dr. Roberto Meléndez y el Sr. Augusto Cornejo de la Sección de Hidrobiología del Museo Nacional de Historia Natural de Chile por las facilidades otorgadas. Finalmente, a tres evaluadores anónimos por sus valiosos comentarios.

\section{Literatura citada}

Anderson E. 1994. Systematics and osteology of the Zoarcidae (Teleostei: Perciformes). Ichthyologic Bulletin JLB Smith Institute of Ichthyology 60, 120 pp.

Anderson E \& A Gosztonyi. 1991. Studies on the Zoarcidae (Teleostei: Perciformes) of the Southern Hemisphere. IV. New records and a new species from the Magellan Province of South America. Ichthyologic Bulletin. JLB Smith Institute of Ichthyology. 55: 1-16.

Anderson E \& V Fedorov. 2004. Family Zoarcidae Swainson 1839, eelpouts. California Academy of Science. Annotated Checklists of Fishes 34: 1-58.

Lloris D \& J Rucabado. 1987. Iluocoetes facali sp. n. (Osteichthyes, Zoarcidae), una nueva especie para la ictiofauna Argentina. Thalassas 5(1): 53-56.

Lloris D \& J Rucabado. 1991. Ictiofauna del Canal Beagle (Tierra del Fuego), aspectos ecológicos y análisis biogeográfico. Publicación Especial del Instituto Español de Oceanografía 8: 1-182.

Morrison E \& G Pequeño. 2003. Primer registro del Zoárcido Crossostomus sobrali (Lloris \& Rucabado, 1987) (Osteichthyes, Zoarcidae) en el Estrecho de Magallanes, Distrito fueguino, Chile. Anales del Instituto de la Patagonia, Punta Arenas (Chile) 31: 105-109.

Ojeda P, F Labra \& A Muñoz. 2000. Biogeographic patterns of Chilean littoral fishes. Revista Chilena de Historia Natural 73: 625-641.

Pequeño G. 1978. Presencia de Notothenia wiltoni Regan, 1913 al norte de los canales de Chiloé, Chile (Teleostomi, Nototheniidae). Noticiero Mensual Museo Nacional de Historia Natural, Chile 22(262): 3-6.

Pequeño G. 1989. Peces de Chile, Lista revisada y comentada. Revista de Biología Marina, Valparaíso 24(2): 1-132.

Recibido el 5 de enero de 2006 y aceptado el 10 de octubre de 2006 